\title{
Effects of Coronary Plaque Wall Properties on the Wall Stress
}

\author{
Kyehan Rhee, Pengsrorn Chhai \\ Myongji University, Department of Mechanical Engineering \\ 38-2 Namdong, Cheoin-gu, Yongin, South Korea \\ khanrhee@mju.ac.kr; pengsrornchhai@yahoo.com
}

\section{Extended Abstract}

Coronary artery disease is one of the major causes of death throughout the world. The rupture of the atherosclerotic plaque is related to the mechanical stress and structural integrity of plaque wall tissues [1]. Disruption of the fibrous cap should be related to the mechanical stress acting on the vessel wall by pulsating arterial pressure as well as hemodynamic wall stress [2]. In order to investigate the effects of plaque wall properties and geometry changes along the stenotic arterial plaque, asymmetric plaque wall models were constructed by skewing the lipid core distribution in the upstream direction.

Mechanical stress in the plaque wall and hemodynamic stress in the lumen were computationally analysed for arterial plaque models. An ideal three dimensional coronary artery with an eccentric stenosis was modelled, and wall stress was analysed considering fluid and structure interactions (FSI). The diameter of the lumen was $3 \mathrm{~mm}$, while the length of the stenosis was $8.2 \mathrm{~mm}$. The stenotic plaque wall is composed of the lipid core and fibrous cap. The luminal height of the throat (minimum lumen cross section) was about $1 \mathrm{~mm}$, and the area reduction was about $36 \%$. The vessel wall thickness was $1 \mathrm{~mm}$, and the minimum thickness of the fibrous cap was about $40 \mu \mathrm{m}$. The 4-node tetrahedral elements were used for computational meshes, and they were fitted with the shape of the vessel lumen and the plaque wall tissue. Finer meshes were used in the plaque cap and the lipid core zone to accommodate abrupt changes in geometry. For all models, the fluid was assumed to be incompressible and Newtonian, with a density of $1,050 \mathrm{~kg} / \mathrm{m}^{3}$ and a viscosity of $0.0035 \mathrm{~kg} / \mathrm{m} \cdot \mathrm{s}$. No slip boundary conditions were used on the walls. The Navier-Stokes equation with arbitrary Lagrangian-Eulerian formulation was used to solve governing equations in the presence of fluid-structure interaction (FSI), and a transient implicit scheme was applied to fluid flow computations.

The result showed that the values of maximum cap stress increased, and its location moved toward the proximal cap as asymmetry increased. The maximum strains in the asymmetric models were also higher compared to those in the symmetric model. The hemodynamic WSS did not change much with longitudinal asymmetry, because the luminal geometry varied only slightly in the asymmetry models. However, the maximum WSS and its spatial gradient, which were believed to be positively related to the endothelium denudation and degradation [3], were higher in the asymmetry models than in the symmetry model. Furthermore, the pressure drop and pressure gradient across the stenosis were higher in the asymmetry models than in the symmetry model. Therefore, higher wall stress and strain, and increased WSS, pressure drops, and gradients of them in asymmetric plaques may provide a more unfavourable biomechanical environment for plaque stability.

\section{Acknowledgments}

This work was supported by the Research Fund (NRF-2017R1A2B4004439).

\section{References}

[1] P. Libby, P., "Mechanisms of acute coronary syndromes," N Engl J Med, vol.369, pp.883-884, 2013.

[2] I. Cicha, A. Wörner, K. Urschel, K. Beronov, M. Goppelt-Struebe, E. Verhoeven, W. Werner Daniel, and C. Garlichs, "Carotid Plaque Vulnerability A Positive Feedback Between Hemodynamic and Biochemical Mechanisms," Stroke, vol.42, pp.3502-3510, 2011.

[3] J. Dolan, J. Kolega, and H. Meng, "High Wall Shear Stress and Spatial Gradients in Vascular Pathology: A Review," Ann Biomed Eng, vol.41, pp.1411-1427, 2013. 